\title{
The Association Between Different Kinds of Exercise and Quality of Life in the Long Term. Results of a Randomized Controlled Trial on the Elderly
}

\author{
Gioia Mura ${ }^{1, *}$, Federica Sancassiani ${ }^{1}$, Gian Mario Migliaccio ${ }^{2}$, GiorgiaCollu $^{2}$ and \\ Mauro Giovanni Carta ${ }^{1}$
}

\author{
${ }^{I}$ Department of Public Health, Clinical and Molecular Medicine, University of Cagliari, Italy; ${ }^{2}$ CONI Italian Olympic \\ Committee, Sardinia, Italy
}

\begin{abstract}
Introduction: Quality of Life (QoL) tends to decrease with age. Exercise has been shown to be effective in improving some psychosocial features related to QoL. We carried out a randomized controlled trial to verify the long-term efficacy of an intensive fitness program versus a lighter program on the QoL of an elderly sample, compared to QoL of a large normative sample. Methods: Participants aged $\geq 65$ years were randomly assigned in a 1:1 fashion either to a vigorous physical activity program group (VAG) or to a postural gymnastic group (PGG). Depressive symptoms were screened by PHQ-9. QoL assessment was done by SF-12. Multivariate analysis of variance (MANOVA) was conducted to test differences between the two groups over time. Comparison with the normative sample was carried out by means of ANOVA 1-way. Results: Both VAG and PGG showed low PHQ9 scores at the beginning and at the end of the trial, indicating the absence of depressive symptoms. At the end of the study, both groups had a higher level of QoL, measured by means of SF-12, than the normative standardized sample. While SF-12 scores for both groups decreased slightly 12 weeks after the end of the trial, only the VAG group maintained significantly-higher scores than those of the normative sample. Conclusions: A vigorous physical activity program group might be associated with better maintenance of results over time as compared to a postural gymnastic program. These results require future confirmation by further studies on large samples.
\end{abstract}

Keywords: Elderly, exercise, physical activity, quality of life.

\section{INTRODUCTION}

A recent community survey showed that Quality of Life (QoL) decreases progressively with age in the general population: the elderly were shown to perceive their QoL as much lower than young people [1].

The presence of chronic inflammatory [2], endocrine [3] and degenerative diseases [4] is associated with reduced QoL; the association of such diseases with mood disorders may be a determining factor amplifying this reduction [2]. Both chronic diseases and their association with mood disorders are common in old age [5].

Physical activity and exercise have recently been shown to improve QoL in elderly people with neurodegenerative diseases [6] and Depressive Disorders [7]. Exercise is associated with better QoL in elderly people with Major Depressive Disorder living in the community [8], and there are strong evidence that physical training improve daily living performance and QoL in frail elderlies long-term care institutionalized [9]. Such an association could be mediated by self-efficacy [10].

*Address correspondence to this author at the Department of Public Health, Clinical and Molecular Medicine, University of Cagliari, Italy; Tel: +39070 6093498; Fax: 0706093498;

E-mail:mura.gioia@virgilio.it
Decreases in sleep and motility are common risk factors for psychological distress in old age, related to poorer QoL [11]. A recent survey revealed a positive chronic association between exercise and time of waking after sleep onset, as well as a small positive acute association between exercise and general sleep quality rating in elderly people [12]. Thus, physical activity not only appears to affect the QoL of older people with chronic diseases, but also physical factors such as sleep, which can be considered a general indicator of wellbeing and a prodromal symptom of physical and mood disorders in people without chronic disease or depression [13].

Moreover, long-term exercise in aging adults has been shown to provide, besides musculoskeletal and cardiovascular positive effects, also a improvement on cognitive functions, such as memory, attention and executive functions [14, 15].

Prevention programs for the elderly, for example against falls, stressed the importance of the effect of physical exercise on psychosocial variables closely related to perceived QoL [16]. It has been suggested that a high perceived QoL is an important motivational factor in preventive programs against chronic vascular diseases in the elderly [17], and it has been shown that long-term physical activity, even in elderlies with chronic diseases and without a clear relationship with a dose-response effect, is related to positive effects on QoL by postponing disability and enhancing independent 
living [18]. Thus, if chronic illnesses and depression can affect QoL, it is also conceivable that a good perceived QoL might be considered a preventive factor against these same disorders in the elderly. A senior who feels well takes better care of himself and eats better, leading to more careful routine clinical examinations.

The present study aims to verify the effect on QoL of an intensive fitness program in an elderly sample without depression as compared to a lighter postural-type fitness program.

\section{METHODS}

\section{Subjects}

Target participants were people aged $\geq 65$ years. The study was carried out as part of the "A Chent'Annos in Salude" (a typical Sardinian saying, which could be translated as "I hope you'll live a hundred years in good health") trial, a prospective population-based study for seniors carried out by the Italian Olympic Committee (CONI) in Sardinia, approved by CONI Sardinia and conducted at the Italian Olympic Committee in Cagliari, Italy, aiming to verify the physiological modifications induced by exercise in the elderly. Participants were recruited by an announcement in a local newspaper, by medical doctors, and by flyers in pharmacies. Approximately 350 people aged 65 or older were invited to participate and were contacted by phone to inform them about the study. More or less 180 people indicated their interest in participating in the study and were added to the list of inclusion-exclusion criteria, established by the doctors on the team.

Inclusion required at least one criteria between sedentary and dysmetabolism according to a medical check-list. In particular, sedentary was defined by an expenditure of energy of less than 1.5 metabolic equivalents (METs), corresponding to remain sitting, reclined, or lying during most of the day [19]; according to the Physical Activity Guidelines for Older Adults, we considered sedentary subjects that performed less than 30 minutes per day of moderate physical activity on five or fewer days per week [20]. According to the American Hearth Association criteria, dysmetabolism was defined by the presence of at least one among: basal glycaemia $\geq 100 \mathrm{mg} / \mathrm{dL}$, systolic blood pressure $\geq 130$ $\mathrm{mmHg}$ and/or diastolic blood pressure $\geq 85 \mathrm{mmHg}$, highdensity lipoprotein plasma cholesterol $\leq 40 \mathrm{mg} / \mathrm{dl}$ (men) or $\leq 50 \mathrm{mg} / \mathrm{dl}$ (women), low-density lipoprotein plasma cholesterol $\geq 160 \mathrm{mg} / \mathrm{dl}$ (men) or $\geq 150 \mathrm{mg} / \mathrm{dl}$ (women), plasma triglycerides $\geq 150 \mathrm{mg} / \mathrm{dl}$, waist circumference $\geq 102 \mathrm{~cm}$ (men) or $\geq 88 \mathrm{~cm}$ (women), or a waist circumference of $\geq 94$ $\mathrm{cm}$ (men) or $\geq 80 \mathrm{~cm}$ (women) in presence of 2 other criteria among the precedents [21].

Exclusion criteria were the presence of: any disease that could be a contraindication to performing a stress test (such as unstable ischemic heart disease, uncontrolled hypertension, severe arrhythmia, valvular heart disease); any osteoarticular pathology incompatible with exercise (severe osteoporosis, acute connective tissue diseases, recent fractures, etc.); neurological diseases or outcomes of debilitating neurological disorders (stroke, dementia, neurodegenerative diseases, etc.); neoplasms in place or disabling outcomes of cancer; moderate to severe anemia $(\mathrm{Hb}<10 \mathrm{mg} / \mathrm{dl})$; obesity with BMI $>35$. Moreover, subjects were included only if they did not have difficulty in reaching the gym where the trial was performed.

All participants selected underwent a detailed medical examination, including anthropological measures; electrocardiogram, blood pressure, and spirometry at rest and under stress test; blood sampling for complete blood count (CBC), glycaemia, total cholesterol, HDL and LDL cholesterol, triglycerides, azotemia, creatinine, cortisol. Written informed consent was obtained. Following this check-up, participants were randomly assigned in a 1:1 fashion to two different groups: VAG (Vigorous Activity Group) and PGG (Postural Gymnastic Group).

\section{Design}

The study was a 12-week randomized controlled trial with fitness sessions 3 times a week, 36 sessions in all, each session lasting 1 hour. Participants continued their regular activity level and dietary habits and were instructed not to engage in any other physical program while participating in the study. The study started in April 2013 (T0) and ended in July 2013 (T1). Data were collected and included in the analysis only for subjects that completed $90 \%$ of the sessions with a maximum absence of 3 non-consecutive days. Twelve weeks later, subjects were again assessed (T2), in order to measure wellbeing after the program and the potential persistence of results.

\section{Fitness Programs}

According to ACSM guidelines [22], Physical Activity (PA) was established as $60 \%$ to $84 \%$ of the Heart Rate Reserve (HRR) and monitored continuously during activity with the heart-rate monitor Polar T31 Coded ${ }^{\mathrm{TM}}$, and also transmitted by a telemetry system $\left(\right.$ Hosand $\left.^{\circledR}\right)$ to the fitness professionals. The individual HRR for each participant was assessed according to the Estimated Maximal Heart Rate Formula [23], where $\mathrm{HR}_{\max }=206.9-(0.67$ x Age). Baseline HR was registered for all participants for three days, in the morning immediately after waking up, and the mean data was utilized.

PA for the VAG consisted of three phases: 1) warm up, up to $10-\mathrm{min}$, up to $60 \%$ of HRR 2) Active phase, 45-min, from $>60 \%$ to $84 \%$ HRR 3 ) cool down, up to $10-\mathrm{min},<60 \%$ of HRR. The Active Phase was designed as a mixture of aerobic and anaerobic exercises. The protocol design integrated drills of "life movements", strength and balance.

Warm Up Phase, up to $60 \%$ of HRR, was designed with slow, dynamic movements using large muscle groups of the lower, middle and upper body, moving through full range of motion. Subjects began with simple walks around the room with various arms movements, including some static and dynamic balance exercises and stretching for principal muscular groups.

Active Phase, from $>60 \%$ to $84 \%$ HRR, was designed with dynamic exercises with and without the use of a platform (step), continuous exercise with gradual increase of the difficulty and the intensity, departing from the walk around the step alternated to the basic step (step up and down from the base with alternate footstep) to reach the alternate lifts with inferior limbs flexes, extended out or flexed behind 
(knee lift, side leg, leg curl), creating some brief and easy sequences to repeat. The last part of the active phase we have been introduced integrated multiple plane exercises for upper and lower limbs using elastic resistances $\left(\right.$ Xertube $^{\circledR}$ ).

Cool Down Phase, $<$ to $60 \%$ of HHR, it included cardio respiratory cool-down exercises followed by relaxation, postural control and spine mobility exercises in prone position (knee-wrist) in support on the platform, exercises of static equilibrium on 4 and 2 supports, with open and closed eyes and with activation of the cores muscles. It also included some post-stretch exercises to return muscles to a pre-exercises length for the muscular groups mostly involved during the workout.

PA for the PGG was designed to maintain the heart rate $\leq$ to $40 \%$ of HRR. It included static and dynamic exercise for postural control and spine mobility exercise in a prone position (knee-wrist) with support on the platform and static equilibrium exercises on 4 and 2 supports, with eyes open and closed and with activation of core muscles.

PA was conducted by fitness professionals with degrees in Sports and Physical Education and continuously monitored by a supervisor responsible for the HRR activity level.

\section{Outcome Measurements}

Depressive symptoms in the two groups were measured at the three steps in the study by means of the Patient Health Questionnaire (PHQ9) [24]. This self-administered questionnaire is a brief, field-tested screening for depression. The assessment is fast and easy, helping clinicians in the diagnosis and treatment of depression. A score between 10 and 15 indicates the presence of relevant depressive symptoms, while a score higher than 15 allows the diagnosis of Major Depressive Disorders. PHQ9 rates were registered at T0, T1 and $\mathrm{T} 2$.

Quality of Life was assessed by the Short Form Health Survey (SF-12) [25]. The SF-12 is a self-evaluation in the previous month of: physical activity, limitations due to physical problems on roles or activities, pain, emotional and general health status, vitality, social activities and mental health. Higher scores on the SF-12 correspond to a better subjective perception of QoL. SF-12 rates were registered at $\mathrm{T} 1$ and $\mathrm{T} 2$.

\section{Statistical Analysis}

Comparison of PHQ9 scores between groups was carried out by means of ANOVA 1-way statistics.

The scores for the SF-12 in the two groups were compared with the normative score for a large national sample standardized by age and sex, drawn from a national survey in Italy [1] by ANOVA 1-way statistics.

To test differences between the two groups (VAG and PGG) on the SF-12 and PHQ9 over time, we conducted a multivariate analysis of variance (MANOVA), with "group" as the between-subjects factor.

\section{Ethical Aspects}

Each subject in the study was identified with a code number not amenable to their name by researchers. Written informed consent was obtained from each participant for data analysis for scientific purposes. The study was approved by the Board of the "Comitato Olimpico Nazionale Italiano" (CONI) of the Sardinia Region.

\section{RESULTS}

21 subjects were assigned to treatment with the VAG (12 males, 9 females), mean age $69.24 \pm 3.94 ; 21$ subjects were assigned to treatment with the PGG (14 males and 7 females), mean age 70.0 5 5.374). In the VAG, 3 subjects reported at least one previous depressive episode, one of them being treated with antidepressant drugs. Two other subjects were receiving benzodiazepines. In the PGG, one person reported at least one previous depressive episode. Another subject was taking antidepressants and benzodiazepines. In the VAG, 11 subjects were receiving pharmacological treatment, in 7 cases multiple drug treatment. In the PGG, 15 subjects were taking medication, in 11 cases multiple drugs. In the VAG, concomitant organic diseases were: hypertension (8 subjects), hypercholesterolemia (3 subjects) and osteoporosis ( 2 subjects); in the PGG, 8 subjects were suffering from hypertension, 5 from diabetes mellitus (type 1 in one case), 4 from hypercholesterolemia, 4 from non-severe heart disease (heart failure, atrial fibrillation, aorto-coronary bypass), 3 from thyroid diseases (post-surgical hypothyroidism, Hashimoto's thyroiditis) and 2 from musculoskeletal diseases (arthritis, osteoporosis).

Two subjects in the VAG group and one in the PGG group dropped out of the study.

Table 1 shows the score on the PHQ9 at T0, T1, and T1. Both the VAG and PGG showed low PHQ9 scores at T0, (ANOVA 1 way: VAG: $\mathrm{F}=0.004 ; \mathrm{p}=0.996$; df $1,57,59$; PGG: $F=; 0.078 ; p=0.925 ;$ df $1,54,56$ ), and scores did not change considering the two factors of time and group (see Table 1. MANOVA PHQ9: time $\mathrm{F}=0.865 ; \mathrm{p}=0.431$; df 2, 32; group $\mathrm{F}=0.284 ; \mathrm{p}=0.598$; df 1,33 ; time ${ }^{*}$ group $\mathrm{F}=1.689$; $\mathrm{p}=0.201 ;$ df 2, 32).

Table 2 shows $\mathrm{SF}-12$ scores at $\mathrm{T} 1$ and $\mathrm{T} 2$ for the $\mathrm{VAG}$, PGG and a normative sample. At the end of the study (T1), the level of QoL measured by means of SF-12 scores was better in both the VAG and the PGG groups than in the normative standardized sample.

SF-12 scores for the two groups decreased slightly 12 weeks after the end of the trial (T2), with no significant differences considering the two factors of time and group (see Table 3. MANOVA SF-12: time $\mathrm{F}=1.386 ; \mathrm{p}=0.247$; df 1, 35; group $\mathrm{F}=0.246 ; \mathrm{p}=0.623$; df 1,35 ; time ${ }^{*}$ group $\mathrm{F}=0.026$; $\mathrm{p}=0.872$; df 1,35$)$. However, the difference between the VAG and the normative sample remained statistically significant (ANOVA 1 way: $\mathrm{F}=5.52 ; \mathrm{p}=0.019 ; \mathrm{df} 1,703,704$ ), while the level of QoL in the PGG group did not differ from the normative sample (ANOVA 1 way: $F=3.10 ; p=0.079$; $d f$ $1,703,704)$.

\section{DISCUSSION}

The results of the study indicate that a sample of elderly people undergoing either a demanding exercise program or a light postural gymnastic program for 12 weeks had a subjective level of appreciation in their QoL, at the end of the trial, 
Table 1. PHQ9 scores in VAG and PGG and multivariate analysis of variance (MANOVA) of PHQ9 for VAG and PGG.

\begin{tabular}{|c|c|c|c|c|}
\hline & VAG & PGG & MANOVA Tests & PHQ-9 \\
\hline \hline PHQ9 T0 & $3.2 \pm 2.9$ & $3.2 \pm 2.9$ & time & $\mathrm{F}=0,865 ; \mathrm{p}=0,431 ; \mathrm{df2}, 32$ \\
\hline PHQ9 T1 & $3.2 \pm 3.4$ & $2.7 \pm 2.7$ & group & $\mathrm{F}=0,284 ; \mathrm{p}=0,598 ; \mathrm{df1}, 33$ \\
\hline PHQ9 T2 & $3.2 \pm 2.8$ & $3.8 \pm 2.8$ & Time * group & $\mathrm{F}=1,689 ; \mathrm{p}=0,201 ; \mathrm{df} 2,32$ \\
\hline
\end{tabular}

PHQ9: Patient Health Questionnaire-9 Items, PGG: Postural Gymnastic Group, VAG: Vigorous Activity Group

higher than the average for individuals of the same age and sex living in the community. The scores for perceived QoL tended to decrease 12 weeks after the end of the trial, although the difference between the scores at the end of the trial and the 12-weeks-later scores did not significantly differ between groups and time. Nevertheless, while the sample of subjects in the PGG group did not differ as compared with the general population, the sample in the VAG group maintained a higher level of QoL than the normative score for a community sample.

Table 2. SF-12 scores comparison between VAG, PGG and a normative sample.

\begin{tabular}{|c|c|c|}
\hline & VAG & PGG \\
\hline \hline SF-12 T1 & $40.1 \pm 4.9$ & $39.1 \pm 5.1$ \\
\hline SF-12 T2 & $39.3 \pm 4.8$ & $38.4 \pm 5.29$ \\
\hline $\begin{array}{c}\text { SF-12 Normative } \\
\text { Italian Sample } \\
\text { (N=685) }\end{array}$ & $35.7 \pm 6.8$ & $35.7 \pm 6.8$ \\
\hline $\begin{array}{c}\text { SF-12T2 vs Normative } \\
\text { SF-12 T3 vs Normative }\end{array}$ & $\begin{array}{c}\mathrm{F}=8.24 ; \mathrm{p}=0.004 ; \\
\text { df } 1,703,704\end{array}$ & $\begin{array}{c}\mathrm{F}=4.91 ; \mathrm{p}=0.019 ; \\
\mathrm{df} 1,703,704\end{array}$ \\
\hline $\begin{array}{c}\mathrm{F}=3.10 ; \mathrm{p}=0.079 ; \\
\mathrm{df} 1,703,704\end{array}$ \\
\hline
\end{tabular}

SF-12: Short Form Health Survey-12 Items, PGG: Postural Gymnastic Group, VAG: Vigorous Activity Group

Table 3. Multivariate analysis of variance (MANOVA) of SF12 for VAG and PGG.

\begin{tabular}{|c|c|}
\hline MANOVA Tests & SF-12 \\
\hline \hline Time & $\mathrm{F}=1,386 \mathrm{p}=0.247 \mathrm{df} 1,35$ \\
\hline Group & $\mathrm{F}=0,246 \mathrm{p}=0,623 \mathrm{df} 1,35$ \\
\hline time * group & $\mathrm{F}=0,026 ; \mathrm{p}=0,872 ; \mathrm{df} 1,35$ \\
\hline
\end{tabular}

SF-12: Short Form Health Survey-12 Items

Previous evidence from the literature stated the effectiveness of exercise in improving mental wellbeing in older people, particularly with a group-based physical activity supervised by trained leaders, with a minimum of two weekly exercise sessions of 45 minutes each [26]. The concept of mental well-being as positive psychological functioning encompassing happiness, life satisfaction and self-esteem
$[27,28]$ is quite different from that represented by Quality of Life, a notion including a more general concept regarding all relevant aspects of life [29]. Our findings suggest that exercise might be associated with a wide range of components of wellbeing, such as subjective satisfaction in life.

Notably, the training group in the present study performed vigorous mixed aerobic/anaerobic physical activity, while most of the trials carried out on healthy elderly people consisted of mild to moderate mixed or aerobic exercise [30]. Nevertheless, the drop-out rate in the VAG group was low $(9.5 \%)$, confirming demanding PA to also be appropriate for elderly people, who are able to adapt and respond to both endurance and strength training [31].

The role of physical activity as a clinical management strategy for depression in the elderly has been widely debated. A recent review of the literature concluded that evidence from high-quality studies was generally positive, but that the effect on depression was minimal [7].

It was argued that participation in physical recreational activities might lead to better QoL outcomes [8], and this effect has been shown as well in patients suffering from treatment-resistant depression [32, 33].

QoL has become an important outcome criterion for psychiatric intervention, particularly in chronic disorders [34]. Patients suffering from either current depressive disorder or milder depressive symptoms have been showed to have worse physical, social, and role functioning, and to feel more physical pain than patients without chronic depressive conditions [35].

Moreover, a high QoL has been shown to be related to positive outcomes in preventive intervention in healthy elderly people, like healthy nutrition and physical activity behavior, recognized as reducing the risk of premature lethality from myocardial infarction, arterial hypertension, colon cancer, type 2 diabetes, osteoporosis and obesity [36]. Recently, it has been highlighted the role of exercise in enhancing metabolic, structural and functional dimensions of the aging brain, determining neuroprotection and preserving cognitive performance [37]. The neurotrophic effect of exercise, which is well known in animals [38], has been shown in humans and hypothesized to be related to the increase of neurotrophic factors, such as the Brain-derived Neurotrophic Factor (BDNF), mainly in the hippocampus [39], involved in memory and mood disorders. This effect of exercise on old people's brain could be an important mediator for the improvement on QoL, and it could protect against a cognitive decline and the loss of autonomy. Conversely, it might be also argued that a high QoL in elderly people could be a strong 
motivation to follow a healthy lifestyle, acting as preventive intervention per se.

Nevertheless, some methodological problems affected the assessment of QoL. There is a possible measurement overlap between QoL and psychopathology, especially depression, which may invalidate research results [34]. Recently, the Longitudinal Investigation of Depression Outcomes (LIDO) trial [40] pointed out that measures of physical and mental health functioning, and QoL showed significant longitudinal associations with measures of depressive symptoms. Moreover, during a current Major Depressive Disorder, mental health-related measurements tended to get worse, while those for physical health changed the least, with measurement of change in QoL in depression being intermediate.

In our study, the result regarding QoL cannot have been influenced by a depressive condition, as it was absent in both samples before, during and after the trial. This independence of results might suggest that the improvement in QoL due to physical exercise may be one of the protective factors against depression in old age.

Elderlies suffering from chronic conditions tend to do less physical activity, thus determining a decrease in motility and functional limitations that could affect overall wellbeing [41]. The issue of loneliness is particularly important for older people, and social contacts might positively influence QoL [42]. We have addressed this issue with group-based intervention.

Moreover, QoL improved in both the VAG and PGG groups after exercise, proving that $\mathrm{QoL}$ is a construct modifiable with health promotion behavior.

\section{LIMITATIONS}

This research was not specifically designed to prove our hypothesis; the sample is small and the association between high QoL and physical activity at the end of the trial might be due to a subject-selection bias, in particular as regards their wellbeing before exercise. QoL was not measured at T0. However, the tendency of scores for the normative sample to decrease to the average of scores for the national SF12 twelve weeks after the end of the trial is a convincing argument regarding the exercise as determinant of the improve in QoL.

\section{CONCLUSIONS}

A 12-week high-intensity program of aerobic/anaerobic physical activity in a sample of subjects aged 65 and over was shown to be associated with higher QoL than a lighter program of a postural type, as compared to the QoL for a normative sample. After 12 weeks of de-training, the group that trained at high intensity maintained a higher level of self-rated QoL as compared to both the low-intensity exercising group and the normative sample. These results require future confirmation by further studies on large samples.

\section{PRACTICAL IMPLICATIONS}

- Quality of Life tends to decrease with age, particularly in presence of chronic diseases and depression.
- Physical activity improves several features related to Quality of Life, such as sleep and motility.

- We show that a high-intensity program of aerobic/anaerobic exercise results associated with a higher Quality of Life in a sample of elderlies, and this finding is maintained after 12 weeks of de-training.

- A high Quality of Life associated with physical activity might be a protective factor against chronic conditions in old age.

\section{CONFLICT OF INTEREST}

The authors confirm that this article content has no conflicts of interest.

\section{ACKNOWLEDGEMENTS}

The "A Chent'Annos in Salude" trial was promoted by CONI Italian Olympic Committee Sardinia, with the approval of the Presidency of the Council of the Province of Cagliari.

\section{REFERENCES}

[1] Carta MG, Aguglia E, Caraci F, et al. Quality of life and urban / rural living: preliminary results of a community survey in Italy. Clin Pract Epidemiol Ment Health 2012; 8: 169-74.

[2] Mura G, Bhat KM, Pisano A, Licci G, Carta MG. Psychiatric symptoms and quality of life in systemic sclerosis. Clin Pract Epidemiol Ment Health 2012; 8: 30-5.

[3] de Ornelas Maia AC, Braga Ade A, Paes F, et al. Comorbidity of depression and anxiety: association with poor quality of life in type 1 and 2 diabetic patients. Clin Pract Epidemiol Ment Health 2013; 9: 136-41.

[4] Carta MG, Mura G, Sorbello O, Farina G, Demelia L. Quality of Life and Psychiatric Symptoms in Wilson's Disease: the Relevance of Bipolar Disorders. Clin Pract Epidemiol Ment Health 2012; 8: 102-9.

[5] Lino VT, Portela MC, Camacho LA, Atie S, Lima MJ. Assessment of social support and its association with depression, self-perceived health and chronic diseases in elderly individuals residing in an area of poverty and social vulnerability in Rio de Janeiro city, Brazil. PLoS One 2013; 8(8): e71712.

[6] Advocat J, Russell G, Enticott J, Hassed C, Hester J, Vandenberg B. The effects of a mindfulness-based lifestyle programme for adults with Parkinson's disease: protocol for a mixed method, randomised two-group control study. BMJ Open 2013; 3(10): e003326.

[7] Mura G, Carta MG. Physical activity in depressed elderly. A systematic review. Clin Pract Epidemiol Ment Health 2013; 9: 125-35.

[8] Patten SB, Williams JWA, Lavorato DH, Bulloch AGM. Recreational physical activity ameliorates some of the negative impact of major depression on health-related quality of life. Front Psychiatry 2013; 4: 22.

[9] Weening-Dijksterhuis E, de Greef MH Scherder EJ, Slaets JP, van der Schans CP. Frail institutionalized older persons: a comprehensive review on physical exercise, physical fitness, activity of daily living, and quality-of-life. Am J Phys Med Rehabil 2011; 90(2): 156-68.

[10] Motl RW, McAuley E. Physical activity, disability, and quality of life in older adults. Phys Med Rehabil Clin N Am 2010; 21(2): 299-308.

[11] Atkins J, Naismith SL, Luscombe GM, Hickie IB. Psychological distress and quality of life in older persons: relative contributions of fixed and modifiable risk factors. BMC Psychiatry 2013; 13(1): 249 .

[12] Dzierzewski JM, Buman MP, Giacobbi PR Jr, et al. Exercise and sleep in community-dwelling older adults: evidence for a reciprocal relationship. J Sleep Res 2014; 23(1): 61-8.

[13] Neikrug AB, Ancoli-Israel S. Sleep disorders in the older adult - a mini-review. Gerontology 2010; 56(2): 181-9. 
[14] Zhao E, Tranovich MJ, Wright VJ. The role of mobility as a protective factor on cognitive functioning in aging adults: a review. Sports Health 2014; 1: 63-9.

[15] Voelcker-Rehage C, Godde B, Staudingr UM. Cardiovascular and coordination training differentially improve cognitive performance and neural processing in older adults. Front Hum Neurosci 2011; 5: 26.

[16] Gschwind YJ, Kressig RW, Lacroix A, Muehlbauer T, Pfenninger B, Granacher U. A best practice fall prevention exercise program to improve balance, strength / power, and psychosocial health in older adults: study protocol for a randomized controlled trial. BMC Geriatr 2013; 13(1): 105.

[17] Michal M, Simon P, Gori T, et al. Psychodynamic Motivation and Training program (PMT) for the secondary prevention in patients with stable coronary heart disease: study protocol for a randomized controlled trial of feasibility and effects. Trials 2013; 14(1): 314 .

[18] Spirduso WW, Cronin DL. Exercise dose-response effects on quality of life and independent living in older adults. Med Sci Sports Exerc 2001; 33(6 Suppl): S598-608.

[19] Owen N, Healy GN, Matthews CE, Dunstan DW. Too much sitting: The population-health science of sedentary behavior. Exerc Sport Sci Rev 2010; 38: 105-13.

[20] Physical Activity Guidelines for Older Adults (65+ years). Available online: https://www.gov.uk/government/uploads/system/ uploads/attachment_data/file/213741/dh_128146.pdf (accessed on 7 March 2014).

[21] Grundy SM, Cleeman JI, Daniels SR, et al. American Heart Association; National Heart, Lung, and Blood Institute. Diagnosis and management of the metabolic syndrome: an American Heart Association/National Heart, Lung, and Blood Institute Scientific Statement. Circulation 2005; 112(17): 2735-52. Epub 2005 Sep 12.

[22] American College of Sports Medicine. ACSM's Guidelines for Exercise Testing and Prescription. Baltimore: Lippincott Williams \& Wilkins 2013 Feb.

[23] Gellish RL, Goslin BR, Olson RE, McDonald A, Russi GD, Moudgil VK. Longitudinal modelling of the relationship between age and maximal heart rate. Med Sci Sports Exerc 2007; 39(5): 822-9.

[24] Gilbody S, Richards D, Brealey S, Hewitt C. Screening for depression in medical settings with the Patient Health Questionnaire (PHQ): a diagnostic meta-analysis. J Gen Intern Med 2007; 22(11): 1596-602.

[25] Ware J Jr, Kosinski M, Keller SD. A 12-Item Short-Form Health Survey: construction of scales and preliminary tests of reliability and validity. Med Care 1996; 34: 220-33.

[26] Windle G, Hughes D, Linck P, Russell I, Woods B. Is exercise effective in promoting mental well-being in older age? A systematic review. Aging Ment Health 2010; 14(6): 652-69.

[27] Vaapio SS, Salminen SJ, Ojanlatva A, Kivela SL. Quality of Life as an outcome of fall prevention interventions among the aged: a systematic review. Eur J Public Health 2009; 19(1): 7-15.
[28] Carta MG, Balestrieri M, Murru A, Hardoy MC. Adjustment Disorder: epidemiology, diagnosis and treatment. Clin Pract Epidemiol Ment Health 2009; 5: 15.

[29] Hendry F, McVittie C. Is quality of life a healthy concept? measuring and understanding life experiences of older people. Qual Health Res 2004; 14: 961.

[30] Mortazavi SS, Mohammad K, Ardebili HE, Beni RD, Mahmoodi M, Keshteli AH. Mental disorder prevention and physical activity in Iranian elderly. Int J Prev Med 2012; 3(Supp11): S64-S72.

[31] [No authors listed]. American College of Sports Medicine Position Stand. Exercise and physical activity for older adults. Med Sci Sports Exerc 1998; 30(6): 992-1008.

[32] Carta MG, Hardoy MC, Pilu A, et al. Improving physical quality of life with group physical activity in the adjunctive treatment of major depressive disorder. Clin Pract Epidemol Ment Health 2008; 4: 1.

[33] Mura G, Moro MF, Patten SB, Carta MG. Exercise as an add-on strategy in the treatment of Major Depressive Disorder. A systematic review. CNS Spectrums. (In Press).

[34] Aigner M, Förster-Streffleur S, Prause W, Freidl M, Weiss M, Bach M. What does the WHOQOL-Bref measure? Measurement overlap between quality of life and depressive symptomatology in chronic somatoform pain disorder. Soc Psychiatry Psychiatr Epidemiol 2006; 41(1): 81-6.

[35] Balestrieri M, Carta MG, Leonetti S, Sebastiani G, Starace F, Bellantuono C. Recognition of depression and appropriateness of antidepressant treatment in Italian primary care. Soc Psychiatry Psychiatr Epidemiol 2004, 39: 171-6.

[36] McNaughton SA, Crawford D, Ball K, Salmon J. Understanding determinants of nutrition, physical activity and quality of life among older adults: Well-being, Eating and Exercise for a Long Life (WELL) study. Health Qual Life Outcomes 2012; 10: 109.

[37] Kirk-Sanchez NJ, McGough EL. Physical exercise and cognitive performance in the elderly: current perspective. Clin Interv Aging 2014; 9: 51-62.

[38] Rasmussen P, Brassard P, Adser H, et al. Evidence for a release of brain-derived neurotrophic factor from the brain during exercise. Exp Physiol 2009; 94(10): 1062-9.

[39] Seifert T, Brassard P, Wissenberg M, et al. Endurance training enhances BDNF release from the human brain. Am J Physiol Regul Integr Comp Physiol 2010; 298(2): R372-R377.

[40] Diehr PH, Derleth AM, McKenna SP, et al. Synchrony of change in depressive symptoms, health status, and quality of life in persons with clinical depression. Health Qual Life Outcomes 2006; 4: 27.

[41] Sawatzky R,Liu-Ambrose T, Miller WC, Marra CA. Physical activity as a mediator of the impact of chronic conditions on quality of life in older adults. Health Qual Life Outcomes 2007; 5: 68.

[42] Farquhar, M. Elderly people's definitions of quality of life. Soc Sci Med 1995; 41(10): 1439-46.

Received: December 17, 2013

Revised: February 06, 2014

Accepted: February 10, 2014

(C) Mura et al.; Licensee Bentham Open.

This is an open access article licensed under the terms of the Creative Commons Attribution Non-Commercial License (http://creativecommons.org/licenses/by-nc/3.0/) which permits unrestricted, non-commercial use, distribution and reproduction in any medium, provided the work is properly cited. 